\title{
Modeling of protein complexes in CASP14 with emphasis on the interaction interface prediction
}

\author{
Justas Dapkunas ${ }^{1}$, Kliment Olechnovič ${ }^{2}$, and Česlovas Venclovas ${ }^{2}$ \\ ${ }^{1}$ Vilnius University \\ ${ }^{2}$ Villnius University
}

May 3, 2021

\begin{abstract}
The goal of CASP experiments is to monitor the progress in the protein structure prediction field. During the 14th CASP edition we aimed to test our capabilities of predicting structures of protein complexes. Our protocol for modeling protein assemblies included both template-based modeling and free docking. Structural templates were identified using sensitive sequence-based searches. If sequence-based searches failed, we performed structure-based template searches using selected CASP server models. In the absence of reliable templates we applied free docking starting from monomers generated by CASP servers. We evaluated and ranked models of protein complexes using an improved version of protein structure quality assessment method, VoroMQA, taking into account both interaction interface and global structure scores. If reliable templates could be identified, generally accurate models of protein assemblies were generated with the exception of an antibody-antigen interaction. The success of free docking mainly depended on the accuracy of initial subunit models and on the scoring of docking solutions. To put our overall results in perspective, we analyzed our performance in the context of other CASP groups. Although the subunits in our assembly models often were not of the top quality, these models had, overall, the best predicted interfaces according to several protein-protein interface accuracy measures. Since we did not use co-evolution-based prediction of inter-chain contacts, we attribute our relative success in predicting interfaces primarily to the emphasis on the interaction interface when modeling and scoring.
\end{abstract}

\section{Introduction}

In recent years the progress in there-dimensional (3D) protein structure prediction was impressive ${ }^{1}$. Application of deep learning-based methods now allows modeling of structures for most of the individual proteins ${ }^{2-4}$. However, the majority of proteins do not function in isolation. They usually perform their functions by interacting with other proteins and assembling into stable or transient protein complexes. Therefore, if we wish to have a detailed understanding of how proteins function, the knowledge of the structures of individual proteins is not sufficient. We need to know the structures of corresponding protein complexes.

The number of possible binary protein-protein interactions is much higher than the number of proteins encoded in genomes, and only a small part of these interactions have already been discovered experimentally ${ }^{5,6}$. Similarly, the number of different structural types of protein complexes is predicted to be much higher than the number of protein folds $\mathrm{s}^{7,8}$. Therefore, the structural modeling of protein-protein interactions represents a more complex problem than the prediction of structures for individual proteins.

Currently, two main methods are available to model the structures of protein complexes. Template-based modeling is based on the observation that homologous proteins often interact in the same way ${ }^{9}$. Thus a 
known structure of a protein complex can serve as a template for modeling homologous protein complexes. If there are no templates, protein-protein docking methods can be used ${ }^{5}$. Docking methods aim to find how proteins interact with each other starting from known structures of individual subunits that can be either solved experimentally or obtained by computational modeling.

The field of protein structure prediction is monitored in the Critical Assessment of Structure Prediction (CASP) experiments that explore every aspect of protein structure modeling ${ }^{1}$. The Critical Assessment of PRedicted Interactions (CAPRI) experiments are devoted to the prediction of the structures for diverse protein complexes ${ }^{10}$. Both CASP and CAPRI are based on blind testing. The participants are given the sequences of proteins, for which structures are solved experimentally but not published (termed "targets"), and then they are asked to provide structural models. Subsequent comparison of models with the experimental structures enables establishing the current state-of-the-art in the field and also objective comparison of different methods. In recent years, CASP and CAPRI experiments are collaborating in the area of structural modeling of protein complexes ${ }^{11,12}$, and a category dedicated to assessment of multimeric proteins has been established in CASP as well ${ }^{13,14}$.

We participated in recent CASP and CAPRI experiments, aiming to test our abilities to predict structures of protein complexes using template-based modeling and free docking ${ }^{15-17}$. Our results demonstrated that there is room for improvement in both of the methods. In template-based modeling, the identification of templates can be enhanced. In docking, the assessment and selection of correct interfaces from thousands of diverse docking models is probably the most important problem. It is also interesting to see how the progress in protein structure prediction influences modeling of protein complexes. At present, it is often possible to generate sufficiently accurate models of individual proteins, but does this help to predict the protein-protein interaction interfaces?

To explore these questions in detail, we participated in the CASP14 experiment, where our group ("Venclovas") performed relatively well, particularly in the interaction interface prediction. In this article we describe our modeling methods and analyze our results in detail aiming to understand what went right, what went wrong and why.

\section{Methods}

\section{Modeling outline}

The outline of our modeling workflow in CASP14 (Figure 1) was largely the same as in CASP13 $3^{17}$, but with several improvements. During the initial step, for every target we attempted to identify multimeric templates for comparative (homology) modeling using sequence- and structure-based search methods. If the identified templates were not reliable, we used template-based docking. Coiled-coil protein structures were predicted using a custom designed procedure. If no templates could be identified, we switched to free docking. In the case of large multisubunit targets, we combined all the methods, employing comparative modeling approach for the parts of the complex for which templates were available followed by docking of the resulting subcomplexes to obtain the full assembly.

\section{Comparative modeling}

The outline of multimeric comparative modeling pipeline is provided in Supplementary Figure S1. Starting with target sequences, we first searched for potential templates using PPI3D ${ }^{18}$ and HHpred ${ }^{19,}{ }^{20}$ web servers. If this step failed to identify reliable templates, we additionally employed structure-based searches. In this case, selected monomeric CASP server models corresponding to the subunits of a target protein complex were used as queries for PDB searches using the DALI server ${ }^{21}$. The aim was to identify multimeric PDB assemblies that could potentially serve as multimeric templates. Once templates were identified using either sequence- or structure-based searches, structural models for the whole protein complex were generated by MODELLER $^{22}$ and its plugin AltMod ${ }^{23}$ using the multi-chain modeling function. 


\section{Template-based docking}

Some targets produced only low-reliability HHpred or DALI results, yet we observed that the identified proteins had similar functional annotations as the CASP targets. If the alignment of CASP server models to these multimeric structures using TM-align ${ }^{24}$ seemed plausible, we simply aligned CASP server models to different chains of the templates and then relaxed the resulting models to remove structural clashes using the same methods as in the case of free docking.

\section{Coiled-coil modeling}

Several targets were predicted to be coiled-coils using MultiCoil2 ${ }^{25}$ and similar sequence analysis tools provided in MPI Bioinformatics Toolkit ${ }^{19}$. Such targets were modeled using a custom designed procedure: structure models were automatically generated by MODELLER/AltMod using the same manually selected coiled-coil template and all possible target-template alignments followed by model selection.

\section{Free docking}

When no templates could be found for protein complexes, free docking of top 5 selected monomeric CASP server models was done by $\mathrm{Hex}^{26}$ for hetero-complexes and $\mathrm{Sam}^{27}$ for homomultimers (Supplementary Figure S2). The resulting models were ranked using global and interaction interface VoroMQA scores as described below. Next, top 100-500 models were relaxed by a very short molecular dynamics simulation using OpenMM software $^{28}$, Amber99SB force field and GBSA-OBC solvation model ${ }^{29}, 30$. The relaxed models were subsequently re-ranked using the same scoring procedure and clustered according to the interface CAD-score ${ }^{31,32}$ values aiming to select a diverse set of models.

\section{Hybrid modeling}

For some large target protein complexes structural templates were available only for some of the subunits or domains. In these cases, a hybrid modeling strategy was used, that is, part of the complex was modeled using comparative modeling whereas remaining subunits were docked to it either by template-based docking using TM-align ${ }^{24}$ or by free docking. In addition to that, a mixture of free docking and template-based models was submitted for several smaller targets that had templates only from structure-based search.

\section{Model selection}

For model selection we used both global structure scores and interaction interface scores. This approach was described previously ${ }^{15,17}$ and implemented in the VoroMQA web server ${ }^{33}$, but for CASP14 we introduced some modifications. These included VoroMQA-dark, a new method for global structure evaluation (see below), and an improved tournament-based ranking algorithm (see Supplementary information for details). When ranking models based on their pairwise comparisons, the algorithm puts more emphasis on the interaction interface pseudo-energy and less emphasis on the global structure score. This is achieved by using a tolerance value when comparing global VoroMQA-dark scores. If the difference between global scores is small, the interface scoring becomes the only decisive factor.

In addition to fully automated scoring methods, models were also evaluated according to constraints obtained from the literature or from the CASP contact prediction servers, if such data were available. All models were visually inspected before submission and manual ranking adjustments were introduced, if necessary.

\section{VoroMQA-dark method for model quality assessment}

VoroMQA-dark is a new model quality assessment method based on the previously published VoroMQA ${ }^{34}$ method (which will be referred to as VoroMQA-light). VoroMQA-dark uses a neural network (NN) trained to predict local (per-residue) CAD-score ${ }^{31}$ values. The global structure score is computed by averaging the predicted local scores. The NN input vector for each residue is computed from the Voronoi tessellation-based contact areas and the corresponding contact potential values from VoroMQA-light. See Supplementary information for more details on VoroMQA-dark. The VoroMQA-dark standalone software is included in the extension 
of the Voronota ${ }^{35}$ package freely available from https://kliment-olechnovic.github.io/voronota/expansion_js/.

\section{Results}

Overview of the results

To analyze our performance in CASP14 we used several accuracy measures designed to evaluate various features of multimeric models. For the overall model evaluation we used QS-score, a distance-based measure of interface accuracy ${ }^{36}$. To make qualitative model accuracy assignments we converted QS-score values to CAPRI-like accuracy categories ${ }^{37}$. Four other scores were used to assess the interface and the overall structure accuracy. Interface Contact Similarity (ICS or F1-score) and Interface Patch Similarity (IPS or Jaccard coefficient) were used to assess contact and interface patch prediction, respectively ${ }^{13}$. Oligomeric IDDT and TM-score were used to assess overall structure accuracy. LDDT is an all-atom superposition-free score $^{38}$, whereas TM-score is based on the rigid body superposition of $\mathrm{C} \alpha$ atoms ${ }^{39},{ }^{40}$. In addition to the above scores reported by the Prediction Center, we also used CAD-score ${ }^{31,32}$ to evaluate the accuracy of both structure and interface accuracy.

The summary of our modeling results based on QS-score is presented in Table 1, whereas the detailed accuracy evaluation of our best models is provided in Supplementary Table S1. As can be seen in Table 1, for 11 targets we identified multimeric templates and used comparative modeling, producing medium or high-accuracy models for 8 of them. In the absence of reliable target-template sequence-structure alignments, we applied template-based docking using TM-align. This approach resulted in models of medium accuracy for 2 targets. Hybrid approaches utilizing both comparative modeling and docking steps were used for 9 targets with relative success. The results of free docking were ranging from completely incorrect to medium accuracy models. The custom modeling procedure that we used for coiled-coils did not produce any reliable models.

\section{Modeling results in the context of other CASP14 groups and automated model selection}

The results, presented in Table 1 and Supplementary Table S1, do not tell much about our relative success. To investigate our performance in the CASP14 context, we compared our results (group "Venclovas") with those of three other top performing groups for models designated as first (model 1). We also included our automatic scoring method ("VoroMQA-select-new") as a virtual group, allowing it to make selections from all CASP14 multimeric models (produced by both automatic servers and human groups). By doing this we aimed to test the effectiveness of our automatic model scoring method in the best possible scenario. For the performance comparison, we used the sum of z-scores of two interface accuracy measures (ICS and IPS) and two global structure accuracy measures (IDDT and TM-score) (Figure 2).

The comparison revealed that different features of our models were predicted with different level of success. According to the accuracy of intersubunit interfaces (ICS and IPS) we achieved the best results. In particular, we were successful in predicting interface patches (IPS), whereas the prediction of specific residue-residue contacts (ICS) was somewhat less successful. On the other hand, the global structure accuracy of our models is not so great compared to other top performing groups. This is especially visible if we consider IDDT, an all-atom score, largely reflecting the accuracy of individual subunits. Interestingly, our automatic model selection method showed relatively strong performance, taking the third position by any of the four scores. Although this method performed worse than our human group on both interface accuracy measures and TM-score, the results according to all-atom accuracy (IDDT) were quite a bit better.

To look at different features in more detail, we examined per-target z-scores. Z-score values were accumulated progressively for targets ordered by the best ICS achieved by any group, which can be interpreted as an estimate of the target difficulty. Figure 3 shows the resulting plots for the models designated as first (model 1). In addition to the data for the same top groups and "VoroMQA-select-new", the plots also include the 
data for the best models provided by any predictor group. The latter curve may be considered as a reference by representing the upper limit of what could have been achieved in CASP14.

Interestingly, the per-target analysis (Fig. 3) revealed that the relative success of different groups was dependent not only on the evaluation measure as seen in Fig. 2, but also on the prediction targets. According to the interface prediction accuracy, our group dominated for most of the targets (Fig. 3A,B). On the other hand, if we consider the global accuracy of models the picture is different. According to TM-score (Fig. 3D) our models are below the state-of-the-art for about half of targets, whereas according to IDDT (Fig. 3C) this is true for nearly all the targets. To see whether our models as assessed by IDDT were indeed significantly inferior to those of other top groups, we examined the cumulative raw values (Supplementary Figure S3). Surprisingly, it turned out that the absolute differences between the groups, especially if evaluated using IDDT (Fig. S3F), are relatively small. This indicates that in most cases subunit structures were of comparable accuracy and that relatively large z-score differences resulted from small structural improvements. The same analysis performed with the CAD-score-based analogs of ICS, IPS and IDDT scores led to similar conclusions (Supplementary Figure S4).

In addition to individual scores, we analyzed their combinations reflecting either the interface prediction accuracy or the accuracy of both the interface and the global structure. We performed this analysis both for models designated as first (Fig. S5) and for the best-of-five models (Fig. S6). The analysis of these combinations has further corroborated above observations on our relative success in the interface prediction and on target-dependent group performance. Interestingly, in the analysis of best-of-five models our automatic selection method (VoroMQA-select-new) was the best according to the interface accuracy (Fig S6A,C) and close to the top according to the combined accuracy (Fig S6B,D). Although having access to all the models VoroMQA-select-new had an important advantage over other groups, the results suggest that this automatic selection procedure is quite robust.

\section{Template-based modeling}

As the structures of protein complexes are often evolutionary conserved ${ }^{9}$, template-based modeling is currently the most reliable method to model them. Straightforward multimeric comparative modeling resulted in medium to high accuracy models for 8 of 11 CASP14 targets. Template-based docking also resulted in medium accuracy models for two targets. Thus, if reliable templates were available the template-based approach worked well for both homomers and heteromers.

Identifying the correct template having the same oligomeric state is the key to successful modeling of protein complexes $^{15-17,36}$. Ambiguous oligomeric state of templates may be the reason why we failed to model T1034, for which we used templates having different oligomeric states.

In CASP14 we had additional examples demonstrating the limitations of template-based modeling for protein complexes. One such example, H1036, represents a trimeric viral protein bound to an antibody. Our models were based on homologous trimer structures bound to antibodies. This resulted in good models of homotrimer interfaces, but the antibody was bound to a completely different epitope (Fig. S7). This incorrectly predicted interface is not surprising bearing in mind the nature of antibody-antigen interactions. The binding site in the antibody (paratope) is formed by hypervariable loop regions, and the antigen binding site (epitope) can be anywhere on the protein surface ${ }^{41}$.

Our results for T1099 show another limitation of template-based approach for protein complexes. This target is a large viral capsid, yet modeling its structure can be reduced to a problem of predicting a homotetramer (T1099v0) having two different interaction interfaces (T1099v1 and T1099v2). Our models contained highaccuracy interface 2, yet the interface 1 was incorrect (Fig. S8). The reason of this failure was the large insertion in the target interface, compared to the template structures.

\section{Hybrid modeling of large targets}

When modeling a large protein complex templates might be available just for some parts of it. During CASP14 this was the case for heteromeric targets H1060 and H1097. For both of these targets more accurate 
models were generated for those parts that had templates.

H1060 was a viral protein complex containing 27 subunits ( 5 homomeric rings bound to each other, A6B3C12D6, Fig. 4). We found templates for all the rings, and generated models of medium accuracy for all of them using either comparative modeling or template-based docking methods. Next, we tried to do template-based docking of the ring models to each other using another viral template, but for such a large complex the docking was complicated. As a result, the accuracy of hetero-complex (H1060v1) model is lower.

Similar situation was observed with hetero-pentamer H1097, where we tried to dock the domains of the $5^{\text {th }}$ subunit to a homology model of a hetero-tetramer albeit with limited success (Supplementary Table S2).

\section{Free docking}

Our free docking results show similar trends as in previous CASP and CAPRI experiments (Fig. 5, Supplementary Table S3) ${ }^{17}$. First, the IPS values are sometimes much higher than ICS. This indicates that the residues mediating protein-protein binding are predicted better than the mutual subunit orientation defining the exact contacts at the interaction interface. Another observation is that the accuracy of subunits matters a lot when docking modeled protein structures. We did not produce any acceptable accuracy models when we were starting from subunit structures of lower accuracy (IDDT $<0.4$ or TM-score $<0.5$ ). It is also important to note that the opposite is not necessarily true. Accurate models of individual subunits do not guarantee accurate docking models.

The most successful docking results were obtained for H1081v0, T1083 (Fig. 6) and T1084. Interestingly, in the cases of T1083 and T1084 the free docking models were better than the template-based models, but the reasons for this are not clear. H1081 was a large target, where two decameric rings had to be docked, and for that we developed a custom procedure. The homology models of decameric rings were aligned on the axis perpendicular to the ring plane and then pushed to each other (using $1 \AA$ steps) and rotated around the axis (using 2 degrees steps), saving every distinct arrangement. Afterwards all obtained models were relaxed, scored and ranked. This custom "two-ring docking" procedure resulted in surprisingly good models.

The modeling of other docking targets was less successful (Fig. 7) illustrating common problems related to the monomer model accuracy and scoring. For example, in the monomer structure of T1054 which we used for docking, the position of N-terminal helix is not compatible with the dimeric structure. The helix is too well packed against the subunit structure, occupying the place of a helix from another subunit in the dimer (Fig. 7A, B). Therefore, it was impossible to obtain a correct docking model starting from such a monomer. Interestingly, when during the post-CASP analysis we repeated the docking experiment using the same monomer model, but without the N-terminal helix, the docking was highly successful (Fig. 7C). Of note, the structure and the oligomeric state of this protein are dynamic. It exists as a monomer, a dimer or a decamer in solution, and the dimer observed in the crystal might represent an intermediate state in decamerization $^{42}$.

In our best model for heterodimeric target H1065 one of the subunits is rotated 180 degrees compared to the experimental structure (Fig. 7E). Again, the interface patch is identified correctly while the interface contacts and subunit orientation are different. Both monomer models are fairly accurate (IDDT $>0.65$, CAD-score $>0.7$, TM-score $>0.8$ ), therefore, their accuracy probably is not the reason of incorrect docking (Fig 7D). However, scoring is really problematic for H1065: both the global and the interface VoroMQA scores of the experimental structure and the model are highly similar (global scores: 0.70 vs. 0.68, interface energy: -354 vs. -388 for target and model, respectively). In other words, had the experimental structure been present together with all the models it would not necessarily have been selected. 


\section{Discussion}

During CASP14 our group used well known methodologies for structural modeling of protein complexes: template-based modeling and rigid body docking. We did not use any deep learning-based inter-chain contacts prediction or refinement using extensive molecular dynamics simulations. Our main aim was accurate prediction of protein-protein interfaces, even if this meant lower global accuracy of models. As the interface accuracy of our models was the best among CASP14 groups predicting protein assemblies (Fig. 2, Fig. 3A,B), it appears that we have coped with this task quite successfully. Probably the main reasons for the successful modeling were (1) effective multimeric template identification by sequence and structure-based methods, (2) model selection procedure, involving improved VoroMQA scoring with more emphasis on the interaction interface, and (3) short molecular dynamics simulations aimed at removing unrealistic geometry and clashes in docking models.

Unlike the interaction interface accuracy, the global accuracy of our models was not the highest (Fig. 2, Fig. 3C,D). This is particularly evident from the close to average values of IDDT, the score that considers all atoms. Most of these lower scores came from template-based models generated using MODELLER/AltMod. When we used CASP server models for docking, IDDT scores were typically higher. This suggests that had we used more advanced modeling techniques ${ }^{43}$, our template-based models might have been of higher global accuracy.

The template-based modeling remains the most accurate method to predict the structures of protein complexes, but the limiting factor for this approach is the detection of structural templates. Typically templates are identified by sequence-based search methods such as BLAST, PSI-BLAST ${ }^{44}$ or HHpred $^{20}$. In CASP14, aiming to expand the set of available templates, we additionally employed structure-based searches. The efficiency of structure-based approach has been greatly increased by the recent advances in protein structure prediction ${ }^{3,4}$. The availability of more accurate models for monomers may be the reason why our structure-based template searches successfully complemented sequence-based searches in CASP14, but less so in CASP $13{ }^{17}$. It is possible that the structure-based template identification for protein complexes may play even a more prominent role in the future.

The template-based modeling of protein complexes represents a more complex problem than the homology modeling of individual proteins. Unlike for monomeric proteins, the modeling of complexes has to deal with additional complications such as the presence of alternative interaction interfaces and differences in stoichiometry of homologous protein complexes ${ }^{15-17,36}$. In CASP14, the modeling of evolutionary non-conserved antibody-antigen interactions was yet another example of a more complex problem. In other cases such as host-pathogen interactions that do not always emerge from long coexistence of species, it might also be hard to apply either template-based or co-evolution-based modeling methods.

When there are no templates and other constraints are lacking, free docking is the only feasible approach to predict the structures of protein complexes. Our CASP14 results support previous observations that docking may be successful only when subunits are sufficiently accurate ${ }^{11,17}$. Thus, recent breakthrough in protein structure prediction might help not only to detect templates for multimeric structures through structure-based searches, but also to expand the applicability of protein docking. However, even if fairly accurate structures of monomers are available, the docking is much better in predicting protein-protein binding sites ${ }^{45,46}$ than the exact mutual arrangement and interface contacts. This has been observed by us both in previous studies ${ }^{17}$ and in CASP14.

CASP14 results showed that our docking workflow still has a lot of room for improvement. With more time and more computational resources devoted for every target, some improvements could be made even while staying in the realm of rigid body docking and keeping our current, admittedly imperfect scoring function: (1) using more different input monomers, and generating even more monomers by modeling domain motions and by remodeling flexible loops and tails; (2) ensuring that the docking software always perform a sufficiently exhaustive sampling of conformations; (3) producing structural variations of each docking solution 
using molecular dynamics or other sampling techniques. These enhancements would allow to explore the conformational space more thoroughly, possibly leading to better results ${ }^{47}$.

Despite limitations of our CASP14 modeling protocol, our strong performance suggests that the prediction of inter-chain contacts using co-evolution and deep learning methods still has little impact on modeling of protein-protein interactions. Why is that? Apparently, there are multiple reasons why inter-chain contact prediction is harder than intra-chain. For example, contact prediction for heteromeric protein complexes requires generating joined multiple sequence alignments. The interacting proteins in the alignment are inferred by genomic distances or by phylogeny ${ }^{48,49}$, as well as selected using automated sequence comparison procedures ${ }^{50}$. However, this significantly reduces the number of sequences in the alignment and does not guarantee correct pairing of proteins. The alignment joining problem is not present for homo-multimers, yet in this case the problem is to distinguish intra-subunit from inter-subunit contacts ${ }^{49}$. So far this problem has been solved by including the monomer structures into the prediction pipeline ${ }^{51}$.

In addition to the issues related to obtaining and analyzing the multiple sequence alignments, training of supervised learning-based methods for contact prediction using the structures of protein complexes may be limited by the availability of experimental structural data. The number of possible protein complexes is believed to outnumber the number of possible protein folds ${ }^{7,8}$, and it is not clear whether known structures represent a significant part of all interaction types ${ }^{8,}{ }^{52}$. Moreover, there are examples of protein-protein interactions such as antibody-antigen or host-pathogen protein interactions, for which principles of co-evolution are hardly applicable.

To conclude, the progress in monomeric protein structure prediction has not yet translated into similar breakthrough in structural modeling of protein complexes. A number of issues of both technical and fundamental nature have to be solved to make a leap in producing reliable structural models of protein interactions, and it will be exciting to see what developments will occur in this research area in the nearest future.

\section{Acknowledgments}

We wish to thank the CASP organizers for making CASP14 happen, the independent assessors for their efforts in evaluating the results and the experimentalists for providing unpublished $3 \mathrm{D}$ structures as prediction targets.

\section{Funding}

Research Council of Lithuania (grants S-MIP-17-60 and S-MIP-21-35).

\section{References}

1. Kryshtafovych A, Schwede T, Topf M, Fidelis K, Moult J. Critical assessment of methods of protein structure prediction (CASP)-Round XIII. Proteins 2019;87(12):1011-1020.

2. Senior AW, Evans R, Jumper J, Kirkpatrick J, Sifre L, Green T, Qin C, Žídek A, Nelson AWR, Bridgland A, Penedones H, Petersen S, Simonyan K, Crossan S, Kohli P, Jones DT, Silver D, Kavukcuoglu K, Hassabis D. Protein structure prediction using multiple deep neural networks in the 13th Critical Assessment of Protein Structure Prediction (CASP13). Proteins 2019;87(12):1141-1148.

3. Senior AW, Evans R, Jumper J, Kirkpatrick J, Sifre L, Green T, Qin C, Žídek A, Nelson AWR, Bridgland A, Penedones H, Petersen S, Simonyan K, Crossan S, Kohli P, Jones DT, Silver D, Kavukcuoglu K, Hassabis D. Improved protein structure prediction using potentials from deep learning. Nature 2020;577(7792):706710. 
4. Yang J, Anishchenko I, Park H, Peng Z, Ovchinnikov S, Baker D. Improved protein structure prediction using predicted interresidue orientations. Proc Natl Acad Sci U S A 2020;117(3):1496-1503.

5. Keskin O, Tuncbag N, Gursoy A. Predicting Protein-Protein Interactions from the Molecular to the Proteome Level. Chem Rev 2016;116(8):4884-4909.

6. Luck K, Kim D-K, Lambourne L, Spirohn K, Begg BE, Bian W, Brignall R, Cafarelli T, Campos-Laborie FJ, Charloteaux B, Choi D, Coté AG, Daley M, Deimling S, Desbuleux A, Dricot A, Gebbia M, Hardy MF, Kishore N, Knapp JJ, Kovács IA, Lemmens I, Mee MW, Mellor JC, Pollis C, Pons C, Richardson AD, Schlabach S, Teeking B, Yadav A, Babor M, Balcha D, Basha O, Bowman-Colin C, Chin S-F, Choi SG, Colabella C, Coppin G, D'Amata C, De Ridder D, De Rouck S, Duran-Frigola M, Ennajdaoui H, Goebels F, Goehring L, Gopal A, Haddad G, Hatchi E, Helmy M, Jacob Y, Kassa Y, Landini S, Li R, Lieshout N van, MacWilliams A, Markey D, Paulson JN, Rangarajan S, Rasla J, Rayhan A, Rolland T, San-Miguel A, Shen Y, Sheykhkarimli D, Sheynkman GM, Simonovsky E, Taşan M, Tejeda A, Tropepe V, Twizere J-C, Wang Y, Weatheritt RJ, Weile J, Xia Y, Yang X, Yeger-Lotem E, Zhong Q, Aloy P, Bader GD, De Las Rivas J, Gaudet S, Hao T, Rak J, Tavernier J, Hill DE, Vidal M, Roth FP, Calderwood MA. A reference map of the human binary protein interactome. Nature 2020;580(7803):402-408.

7. Aloy P, Russell RB. Ten thousand interactions for the molecular biologist. Nat Biotechnol 2004;22(10):1317-1321.

8. Garma L, Mukherjee S, Mitra P, Zhang Y. How many protein-protein interactions types exist in nature? PLoS One 2012;7(6):e38913.

9. Aloy P, Ceulemans H, Stark A, Russell RB. The relationship between sequence and interaction divergence in proteins. J Mol Biol 2003;332(5):989-998.

10. Lensink MF, Nadzirin N, Velankar S, Wodak SJ. Modeling protein-protein, protein-peptide, and proteinoligosaccharide complexes: CAPRI 7th edition. Proteins 2020;88(8):916-938.

11. Lensink MF, Velankar S, Kryshtafovych A, Huang S-Y, Schneidman-Duhovny D, Sali A, Segura J, Fernandez-Fuentes N, Viswanath S, Elber R, Grudinin S, Popov P, Neveu E, Lee H, Baek M, Park S, Heo L, Rie Lee G, Seok C, Qin S, Zhou H-X, Ritchie DW, Maigret B, Devignes M-D, Ghoorah A, Torchala M, Chaleil RAG, Bates PA, Ben-Zeev E, Eisenstein M, Negi SS, Weng Z, Vreven T, Pierce BG, Borrman TM, Yu J, Ochsenbein F, Guerois R, Vangone A, Rodrigues JPGLM, Zundert G van, Nellen M, Xue L, Karaca E, Melquiond ASJ, Visscher K, Kastritis PL, Bonvin AMJJ, Xu X, Qiu L, Yan C, Li J, Ma Z, Cheng J, Zou X, Shen Y, Peterson LX, Kim H-R, Roy A, Han X, Esquivel-Rodriguez J, Kihara D, Yu X, Bruce NJ, Fuller JC, Wade RC, Anishchenko I, Kundrotas PJ, Vakser IA, Imai K, Yamada K, Oda T, Nakamura T, Tomii K, Pallara C, Romero-Durana M, Jiménez-García B, Moal IH, Férnandez-Recio J, Joung JY, Kim JY, Joo K, Lee J, Kozakov D, Vajda S, Mottarella S, Hall DR, Beglov D, Mamonov A, Xia B, Bohnuud T, Del Carpio CA, Ichiishi E, Marze N, Kuroda D, Roy Burman SS, Gray JJ, Chermak E, Cavallo L, Oliva R, Tovchigrechko A, Wodak SJ. Prediction of homoprotein and heteroprotein complexes by protein docking and template-based modeling: A CASP-CAPRI experiment. Proteins 2016;84 Suppl 1:323-348.

12. Lensink MF, Brysbaert G, Nadzirin N, Velankar S, Chaleil RAG, Gerguri T, Bates PA, Laine E, Carbone A, Grudinin S, Kong R, Liu R-R, Xu X-M, Shi H, Chang S, Eisenstein M, Karczynska A, Czaplewski C, Lubecka E, Lipska A, Krupa P, Mozolewska M, Golon E, Samsonov S, Liwo A, Crivelli S, Pagès G, Karasikov M, Kadukova M, Yan Y, Huang S-Y, Rosell M, Rodríguez-Lumbreras LA, Romero-Durana M, Díaz-Bueno L, Fernandez-Recio J, Christoffer C, Terashi G, Shin W-H, Aderinwale T, Maddhuri Venkata Subraman SR, Kihara D, Kozakov D, Vajda S, Porter K, Padhorny D, Desta I, Beglov D, Ignatov M, Kotelnikov S, Moal IH, Ritchie DW, Chauvot de Beauchêne I, Maigret B, Devignes M-D, Ruiz Echartea ME, Barradas-Bautista D, Cao Z, Cavallo L, Oliva R, Cao Y, Shen Y, Baek M, Park T, Woo H, Seok C, Braitbard M, Bitton L, Scheidman-Duhovny D, Dapkūnas J, Olechnovič K, Venclovas C̆, Kundrotas PJ, Belkin S, Chakravarty D, Badal VD, Vakser IA, Vreven T, Vangaveti S, Borrman T, Weng Z, Guest JD, Gowthaman R, Pierce BG, Xu X, Duan R, Qiu L, Hou J, Ryan Merideth B, Ma Z, Cheng J, Zou X, Koukos PI, Roel-Touris J, 
Ambrosetti F, Geng C, Schaarschmidt J, Trellet ME, Melquiond ASJ, Xue L, Jiménez-García B, Noort CW van, Honorato RV, Bonvin AMJJ, Wodak SJ. Blind prediction of homo- and hetero-protein complexes: The CASP13-CAPRI experiment. Proteins 2019;87(12):1200-1221.

13. Lafita A, Bliven S, Kryshtafovych A, Bertoni M, Monastyrskyy B, Duarte JM, Schwede T, Capitani G. Assessment of protein assembly prediction in CASP12. Proteins 2018;86 Suppl 1:247-256.

14. Guzenko D, Lafita A, Monastyrskyy B, Kryshtafovych A, Duarte JM. Assessment of protein assembly prediction in CASP13. Proteins 2019;87(12):1190-1199.

15. Dapkūnas J, Olechnovič K, Venclovas Č. Modeling of protein complexes in CAPRI Round 37 using template-based approach combined with model selection. Proteins 2018;86 Suppl 1:292-301.

16. Dapkūnas J, Kairys V, Olechnovič K, Venclovas Č. Template-based modeling of diverse protein interactions in CAPRI rounds 38-45. Proteins 2020;88(8):939-947.

17. Dapkūnas J, Olechnovič K, Venclovas Č. Structural modeling of protein complexes: Current capabilities and challenges. Proteins 2019;87(12):1222-1232.

18. Dapkūnas J, Timinskas A, Olechnovič K, Margelevičius M, Dičiūnas R, Venclovas Č. The PPI3D web server for searching, analyzing and modeling protein-protein interactions in the context of 3D structures. Bioinformatics 2017;33(6):935-937.

19. Zimmermann L, Stephens A, Nam S-Z, Rau D, Kübler J, Lozajic M, Gabler F, Söding J, Lupas AN, Alva V. A Completely Reimplemented MPI Bioinformatics Toolkit with a New HHpred Server at its Core. J Mol Biol 2018;430(15):2237-2243.

20. Söding J. Protein homology detection by HMM-HMM comparison. Bioinformatics 2005;21(7):951-960.

21. Holm L. DALI and the persistence of protein shape. Protein Sci 2020;29(1):128-140.

22. Šali A, Blundell TL. Comparative Protein Modelling by Satisfaction of Spatial Restraints. J Mol Biol 1993;234(3):779-815.

23. Janson G, Grottesi A, Pietrosanto M, Ausiello G, Guarguaglini G, Paiardini A. Revisiting the "satisfaction of spatial restraints" approach of MODELLER for protein homology modeling. PLoS Comput Biol 2019;15(12):e1007219.

24. Zhang Y, Skolnick J. TM-align: a protein structure alignment algorithm based on the TM-score. Nucleic Acids Res 2005;33(7):2302-2309.

25. Trigg J, Gutwin K, Keating AE, Berger B. Multicoil2: predicting coiled coils and their oligomerization states from sequence in the twilight zone. PLoS One 2011;6(8):e23519.

26. Ritchie DW, Kemp GJ. Protein docking using spherical polar Fourier correlations. Proteins 2000;39(2):178-194.

27. Ritchie DW, Grudinin S. Spherical polar Fourier assembly of protein complexes with arbitrary point group symmetry. J Appl Cryst 2016;49(1):158-167.

28. Eastman P, Swails J, Chodera JD, McGibbon RT, Zhao Y, Beauchamp KA, Wang L-P, Simmonett AC, Harrigan MP, Stern CD, Wiewiora RP, Brooks BR, Pande VS. OpenMM 7: Rapid development of high performance algorithms for molecular dynamics. PLoS Comput Biol 2017;13(7):e1005659.

29. Hornak V, Abel R, Okur A, Strockbine B, Roitberg A, Simmerling C. Comparison of multiple Amber force fields and development of improved protein backbone parameters. Proteins 2006;65(3):712-725.

30. Onufriev A, Bashford D, Case DA. Exploring protein native states and large-scale conformational changes with a modified generalized born model. Proteins 2004;55(2):383-394. 
31. Olechnovič K, Kulberkytė E, Venclovas Č. CAD-score: a new contact area difference-based function for evaluation of protein structural models. Proteins 2013;81(1):149-162.

32. Olechnovič K, Venclovas Č. Contact Area-Based Structural Analysis of Proteins and Their Complexes Using CAD-Score. Methods Mol Biol 2020;2112:75-90.

33. Olechnovič K, Venclovas Č. VoroMQA web server for assessing three-dimensional structures of proteins and protein complexes. Nucleic Acids Res 2019;47(W1):W437-W442.

34. Olechnovič K, Venclovas Č. VoroMQA: Assessment of protein structure quality using interatomic contact areas. Proteins 2017;85(6):1131-1145.

35. Olechnovič K, Venclovas Č. Voronota: A fast and reliable tool for computing the vertices of the Voronoi diagram of atomic balls. J Comput Chem 2014;35(8):672-681.

36. Bertoni M, Kiefer F, Biasini M, Bordoli L, Schwede T. Modeling protein quaternary structure of homoand hetero-oligomers beyond binary interactions by homology. Sci Rep 2017;7(1):10480.

37. Méndez R, Leplae R, De Maria L, Wodak SJ. Assessment of blind predictions of protein-protein interactions: current status of docking methods. Proteins 2003;52(1):51-67.

38. Mariani V, Biasini M, Barbato A, Schwede T. IDDT: a local superposition-free score for comparing protein structures and models using distance difference tests. Bioinformatics 2013;29(21):2722-2728.

39. Zhang Y, Skolnick J. Scoring function for automated assessment of protein structure template quality. Proteins 2004;57(4):702-710.

40. Mukherjee S, Zhang Y. MM-align: a quick algorithm for aligning multiple-chain protein complex structures using iterative dynamic programming. Nucleic Acids Res 2009;37(11):e83.

41. Sela-Culang I, Kunik V, Ofran Y. The structural basis of antibody-antigen recognition. Front Immunol 2013;4:302.

42. Grinter R, Morris FC, Dunstan RA, Leung PM, Belousoff M, Gunasinghe SD, Beckham S, Peleg AY, Greening C, Li J, Heinz E, Lithgow T. BonA from Acinetobacter baumannii forms a divisome-localized decamer that supports outer envelope function. bioRxiv 2020:2020.09.01.278697.

43. Song Y, DiMaio F, Wang RY-R, Kim D, Miles C, Brunette T, Thompson J, Baker D. High-resolution comparative modeling with RosettaCM. Structure 2013;21(10):1735-1742.

44. Altschul SF, Madden TL, Schäffer AA, Zhang J, Zhang Z, Miller W, Lipman DJ. Gapped BLAST and PSI-BLAST: a new generation of protein database search programs. Nucleic Acids Res 1997;25(17):33893402.

45. Fernández-Recio J, Totrov M, Abagyan R. Identification of protein-protein interaction sites from docking energy landscapes. J Mol Biol 2004;335(3):843-865.

46. Viswanathan R, Fajardo E, Steinberg G, Haller M, Fiser A. Protein-protein binding supersites. PLoS Comput Biol 2019;15(1):e1006704.

47. Kurkcuoglu Z, Bonvin AMJJ. Pre- and post-docking sampling of conformational changes using ClustENM and HADDOCK for protein-protein and protein-DNA systems. Proteins 2020;88(2):292-306.

48. Zeng H, Wang S, Zhou T, Zhao F, Li X, Wu Q, Xu J. ComplexContact: a web server for inter-protein contact prediction using deep learning. Nucleic Acids Res 2018;46(W1):W432-W437.

49. Szurmant H, Weigt M. Inter-residue, inter-protein and inter-family coevolution: bridging the scales. Curr Opin Struct Biol 2018;50:26-32.

50. Faure G, Andreani J, Guerois R. InterEvol database: exploring the structure and evolution of protein complex interfaces. Nucleic Acids Res 2012;40(Database issue):D847-856. 
51. Uguzzoni G, John Lovis S, Oteri F, Schug A, Szurmant H, Weigt M. Large-scale identification of coevolution signals across homo-oligomeric protein interfaces by direct coupling analysis. Proc Natl Acad Sci U S A 2017;114(13):E2662-E2671.

52. Ahnert SE, Marsh JA, Hernández H, Robinson CV, Teichmann SA. Principles of assembly reveal a periodic table of protein complexes. Science 2015;350(6266):aaa2245.

\section{Tables}

Table 1. Summary of our CASP14 assembly modeling results

\begin{tabular}{llll}
\hline Modeling strategy & Number of targets & Accuracy category of our best model & Accuracy category of our best mode \\
\hline & & High (QS-score [?] 0.7) & Medium $(0.3$ [?] QS-score $<0.7)$ \\
Comparative modeling & 11 & 1 & 7 \\
Template-based docking & 2 & 0 & 2 \\
Hybrid & 9 & 1 & 3 \\
Free docking & 5 & 0 & 1 \\
Coiled-coil modeling & 2 & 0 & 0 \\
Total & 29 & 2 & 13 \\
\hline
\end{tabular}

\section{Figure legends}

Figure 1. Summary of modeling workflow in CASP14.

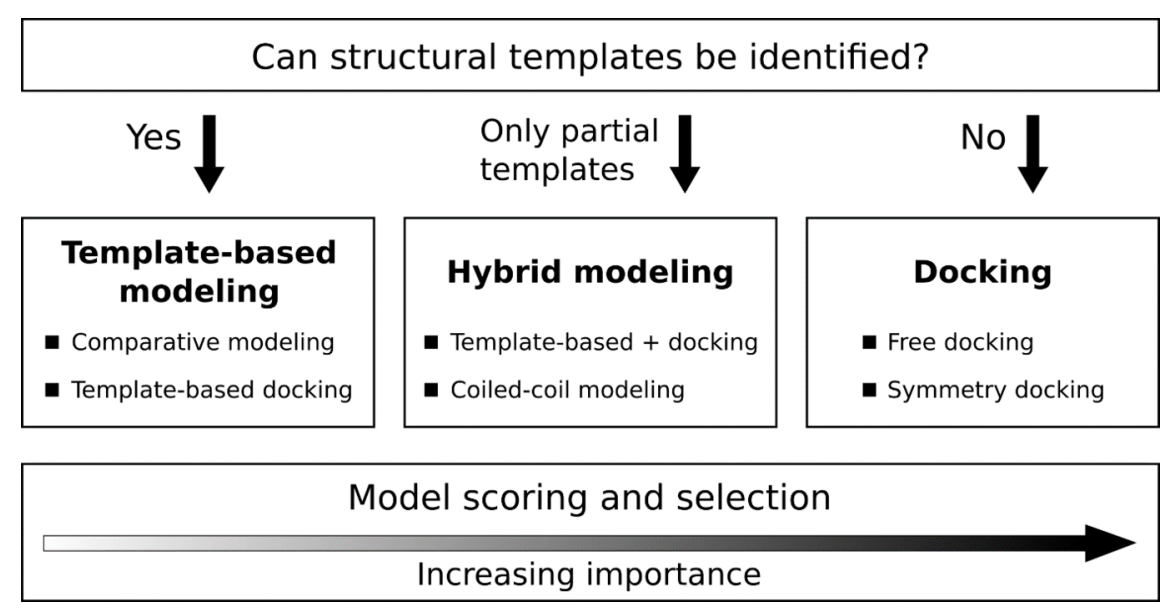

Figure 2 . Comparison of results of our group ("Venclovas") and our automated model selection method ("VoroMQA-select-new") with other top performing CASP14 groups. 


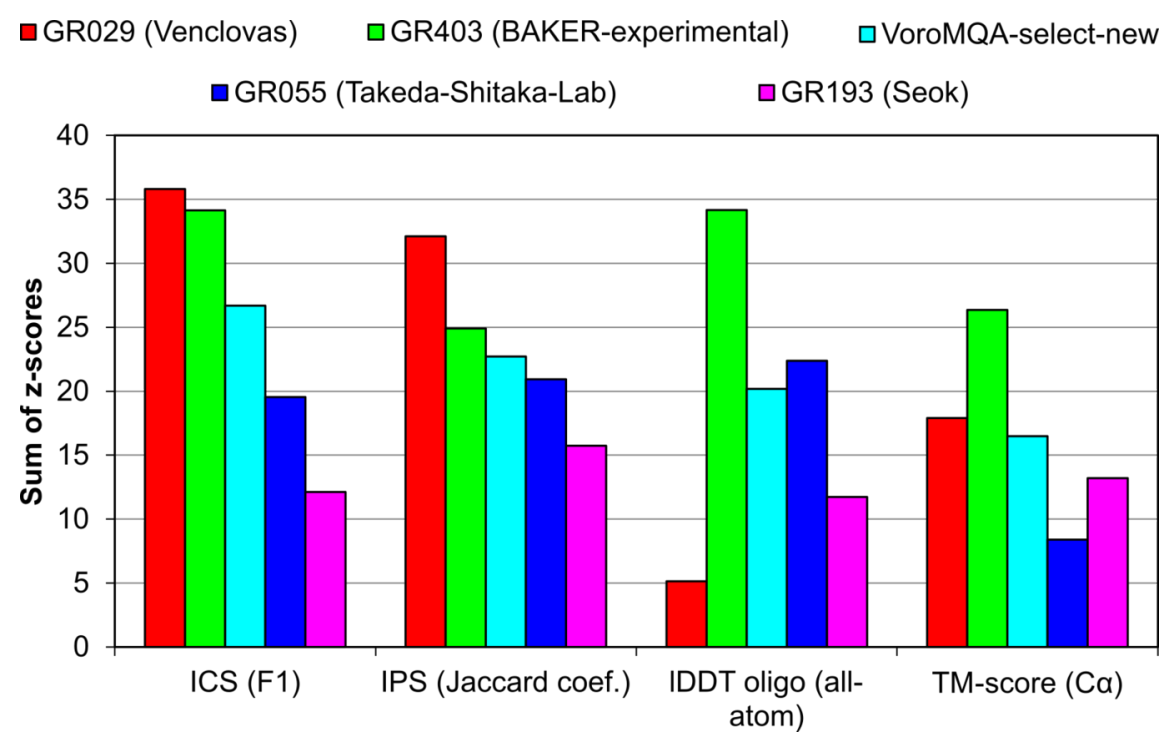

Figure 3 . Cumulative z-score values of the models designated as first, targets were ordered by the maximum achieved ICS score.
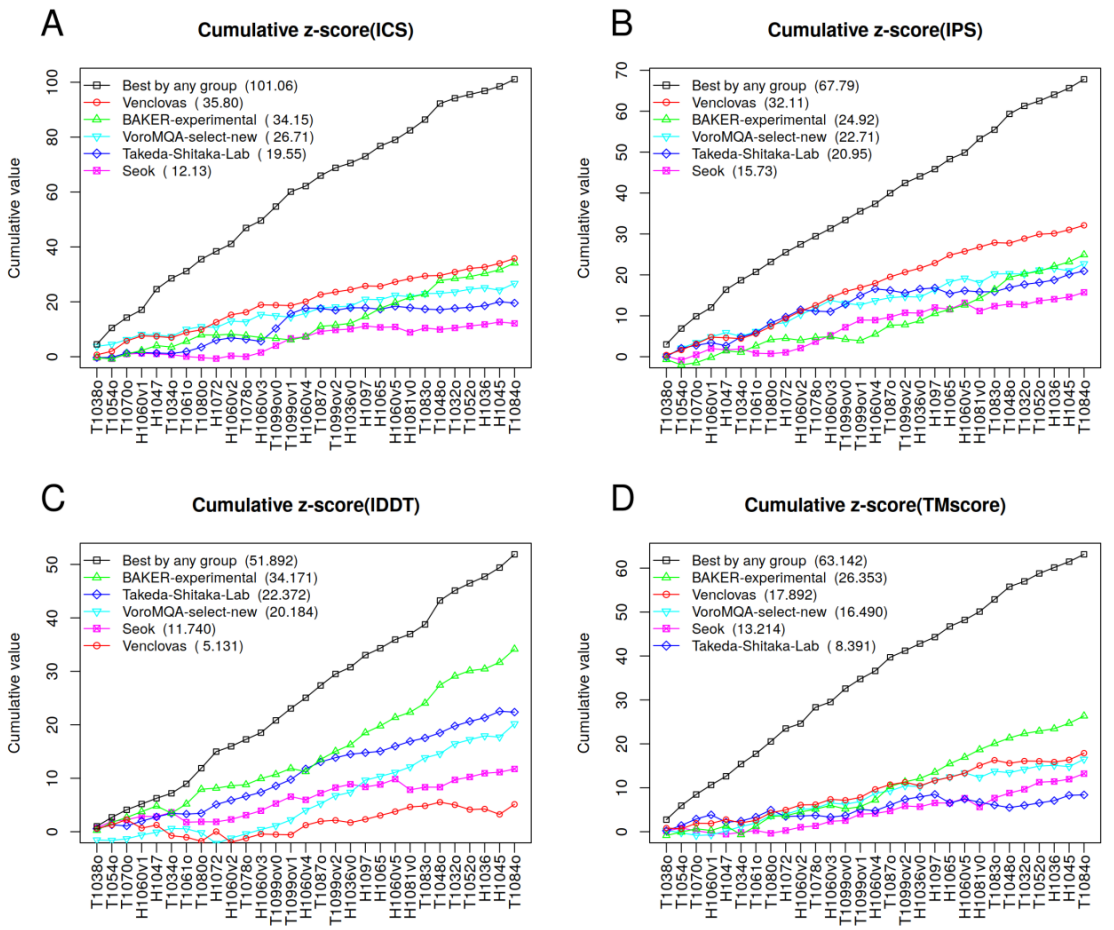

Figure 4. Modeling of H1060. In the images of subcomplexes v1-v5 the target structure is shown in gray and the model structure is colored. 

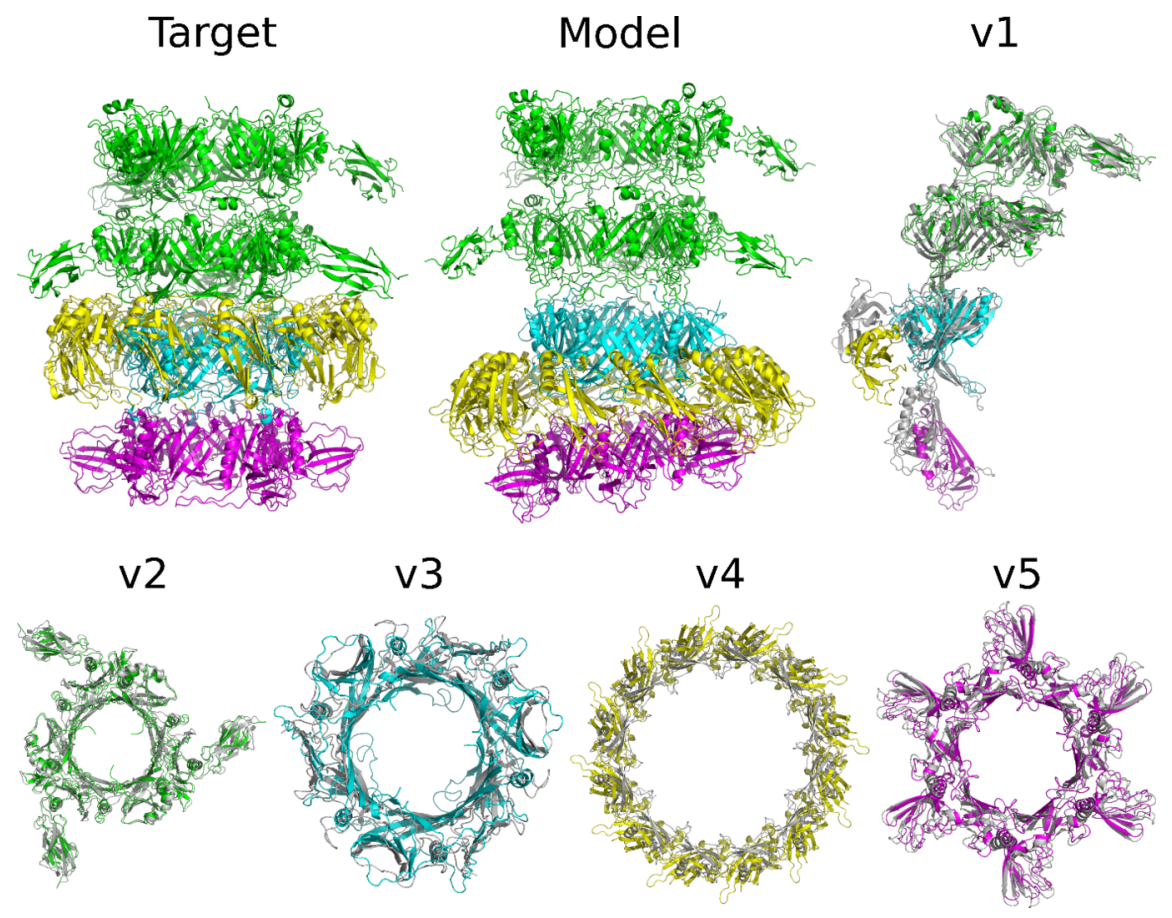

Figure 5 . Dependence of the predicted interface accuracy on the subunit accuracy in our free docking models having the largest ICS or IPS scores.
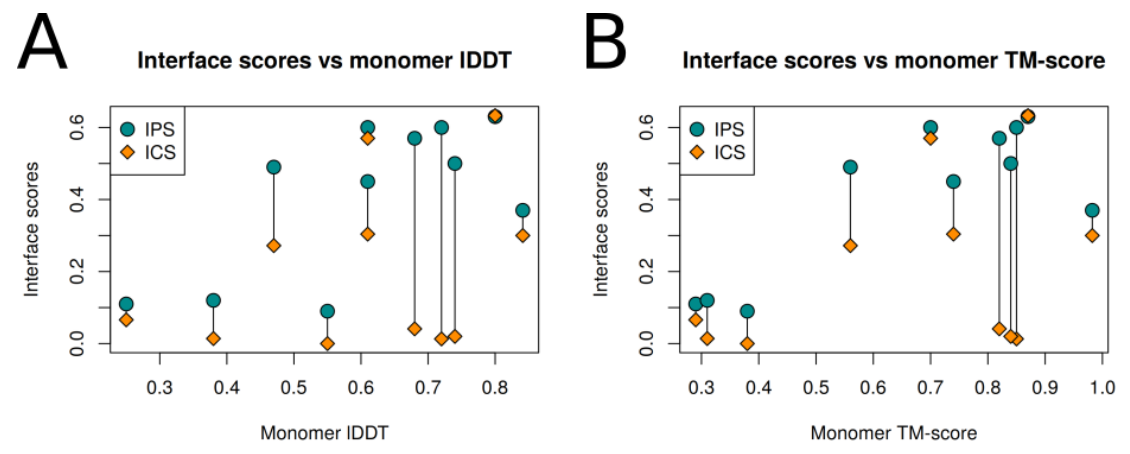

Figure 6 . Successful docking models for H1081 and T1083.

$\mathrm{H} 1081$

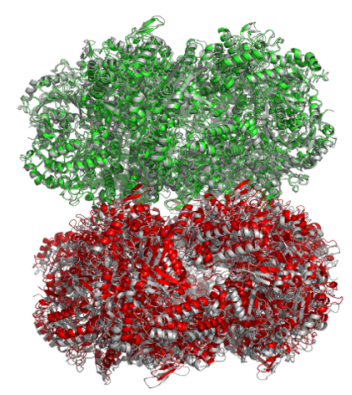

H1081v0

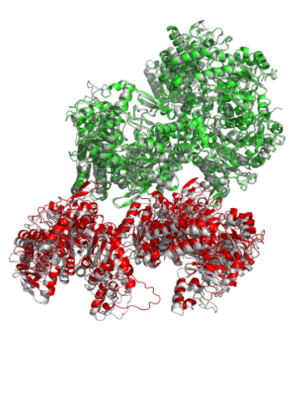

T1083

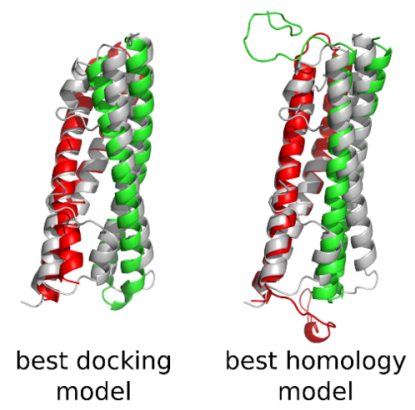


Figure 7 . Free docking results for T1054 and H1065; A, a monomer model of T1054 has additional folded helix in the N-terminus (blue, encircled); B, a dimer model (interface CAD-score 0, binding site CAD-score 0.34), in which the N-terminal helix (encircled) occupies the place of another subunit; C, a dimer model (interface CAD-score 0.33, binding site CAD-score 0.58), in which a monomer without $\mathrm{N}$-terminus was used for docking; D, monomer models of H1065; E, interface patch is predicted better than the interface contacts for H1065 because subunit 2 (red) is rotated $\sim 180$ degrees in the best model of H1065. Target structures are grey, model structures are colored in all images.
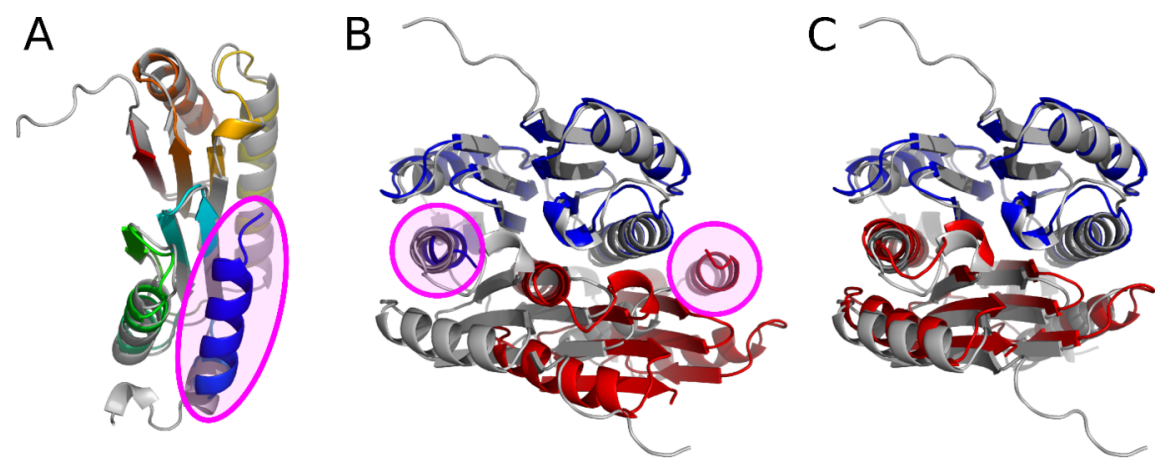

D

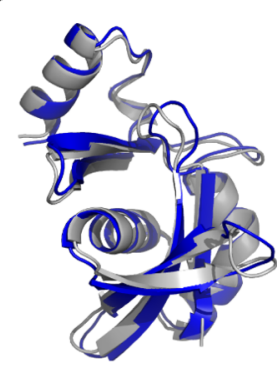

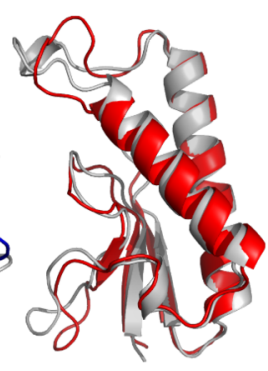

$\mathrm{E}$

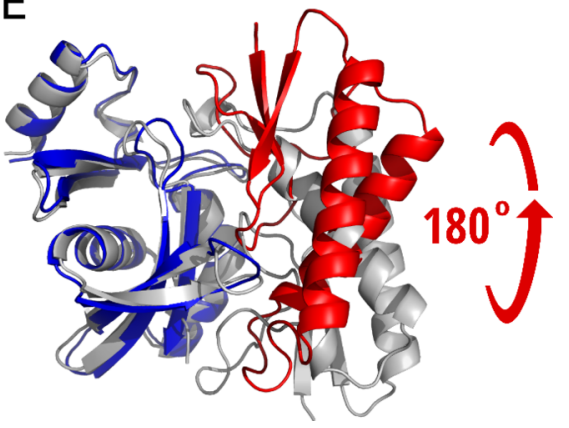

